OPEN ACCESS

Edited by:

Constantin Mircioiu, Carol Davila University of Medicine and

Pharmacy, Romania

Reviewed by: Satish Kumar Bedada, Sanofi India Limited, India

Fawad Rasool,

Bahauddin Zakariya University,

Pakistan

*Correspondence:

Bing Han

hbshcn@163.com

Xiaoqiang Xiang

xiangxq@fudan.edu.cn

Specialty section:

This article was submitted to Drug Metabolism and Transport, a section of the journal Frontiers in

Pharmacology

Received: 22 September 2021

Accepted: 20 October 2021

Published: 22 November 2021

Citation:

Liu H, Yu Y, Guo N, Wang X, Han B and Xiang $X$ (2021) Application of

Physiologically Based

Pharmacokinetic Modeling to Evaluate the Drug-Drug and Drug-Disease

Interactions of Apatinib.

Front. Pharmacol. 12:780937.

doi: 10.3389/fphar.2021.780937

\section{Application of Physiologically Based Pharmacokinetic Modeling to Evaluate the Drug-Drug and Drug-Disease Interactions of Apatinib}

\author{
Hongrui Liu ${ }^{1}$, Yiqun $\mathrm{Yu}^{1}$, Nan Guo ${ }^{1}$, Xiaojuan Wang ${ }^{1}$, Bing Han ${ }^{1 *}$ and Xiaoqiang Xiang ${ }^{2 *}$ \\ ${ }^{1}$ Department of Pharmacy, Minhang Hospital, Fudan University, Shanghai, China, ${ }^{2}$ Department of Clinical Pharmacy, School of \\ Pharmacy, Fudan University, Shanghai, China
}

Aim: Apatinib is an orally administered vascular epidermal growth factor receptor (VEGFR)-tyrosine kinase inhibitors approved for the treatment of advanced gastric adenocarcinoma or gastric esophageal junction adenocarcinoma. Apatinib is predominantly metabolized by CYP3A4/5, followed by CYP2D6. The present study aimed to evaluate the potential drug-drug interaction (DDI) and drug-disease interaction (DDZI) risks of apatinib in Chinese volunteers.

Methods: Modeling and simulation were conducted using Simcyp Simulator. The input parameters required for modeling were obtained from literature research or experiments. Then, the developed physiologically based pharmacokinetic (PBPK) models were applied to evaluate single-dose DDI potential in Chinese healthy volunteers with weak and moderate CYP3A inhibitors, strong CYP2D6 inhibitors, as well as CYP3A4 inducers. The DDZI potential was also predicted in patients with hepatic or renal impairment.

Results: The developed PBPK models accurately assessed apatinib pharmacokinetics following single-dose administration in Chinese healthy volunteers and cancer patients. The DDI simulation showed 2-4-fold changes in apatinib exposures by moderate CYP3A4 inhibitors and CYP3A4 inducers. A moderate increase of apatinib exposure (1.25-2-fold) was found with strong CYP2D6 inhibitor. In the DDZI simulation with hepatic impairment, the AUC of apatinib was significantly increased by 2.25 -fold and 3.04-fold for Child-Pugh B and Child-Pugh C, respectively, with slightly decreased $C_{\max }$ by 1.54 and 1.67 -fold, respectively.

Conclusion: The PBPK models developed in the present study would be highly beneficial to quantitatively predict the pharmacokinetic changes of apatinib under different circumstances, which might be difficult to evaluate clinically, so as to avoid some risks in advance.

Keywords: apatinib, tyrosine kinase inhibitors (TKIs), physiologically based pharmacokinetic (PBPK) models, drug-drug interaction (DDI), drug-disease interaction (DDZI) 


\section{INTRODUCTION}

Drug-drug interaction (DDI) is a phenomenon of modification of pharmacological activity of one drug by another coadministered drug (Min and Bae, 2017; Rekić et al., 2017). Pharmacokinetic (PK) DDIs are the most common type and happens when the absorption, distribution, metabolism, or excretion (ADME) of the substrates are affected by coadministered drugs, resulting in an increased or decreased exposure of the substrates (Min and Bae, 2017; Chung and Kesisoglou, 2018; Wu et al., 2020). Understanding how certain co-administered drugs affect the exposure changes of substrate is essential for drug development and application (Zhang et al., 2012). The Japanese Medicines and Medical Devices Administration (PMDA), European Medicines Agency (EMA), and U.S. Food and Drug Administration (FDA) have issued separate DDI guidelines, emphasizing the predictive use of comprehensive mechanism methods, such as physiologically based pharmacokinetic (PBPK) models, for quantitative evaluation of potential DDI risk in different periods of drug discovery and development (Committee for Human Medicinal Products, 2012; Drug Interaction Studies, 2012; Drug Interaction Studies, 2014). Due to the fact that most drugs are primarily eliminated via liver and kidneys, the impairment of organ function of liver and kidney is one of the important intrinsic factors for regulating drug exposure, that is, drug-disease interaction (DDZI). Consequently, the FDA and EMA also published guidelines to assess the impact of hepatic and/or renal impairment (HIs and RIs, respectively) on the pharmacokinetics of drugs, providing the dosing and labeling recommendations (Guidance for Industry, 2003; Guidance for Industry, 2010; Committee for Human Medicinal Products, 2014; Committee for Human Medicinal Products, 2015).

Apatinib is an oral small-molecule tyrosine kinase inhibitor (TKI), which can competitively bind to the tyrosine ATP binding site in vascular endothelial growth factor receptor (VEGFR), selectively inhibit the activity of VEGFR tyrosine kinases, and block the downstream signaling, therefore suppressing the tumor angiogenesis (Roviello et al., 2016; Geng et al., 2018; Meng et al., 2020). Apatinib shows good oral bioavailability and tolerance in humans. In 2014, apatinib was approved by the National Medical Products Administration for the treatment of advanced gastric adenocarcinoma or gastric esophageal junction adenocarcinoma (Roviello et al., 2016; Geng et al., 2018). Clinical trials have shown that apatinib has certain objective remission rate and survival benefit in the treatment of a variety of malignant tumors, i.e., breast cancer, non-small cell lung cancer, hepatocellular carcinoma, and colorectal cancer (Zhang, 2015; Xue et al., 2018; Fathi Maroufi et al., 2020). In vitro metabolism studies showed that apatinib was the substrate of P450 enzymes, which was mainly metabolized via CYP3A4/5 and, to a lesser extent, by CYP2D6 (Ding et al., 2013). The plasma exposure of apatinib was reported to be significantly affected by co-administration with itraconazole $\left(\mathrm{AUC}_{0-\mathrm{t}}\right.$ increased by $\left.75 \%\right)$ or rifampin $\left(\mathrm{AUC}_{0-\mathrm{t}}\right.$ decreased by $83 \%$ ) in humans, indicating the high potential of DDIs by affecting drug metabolism enzymes for apatinib (Liu et al., 2018). However, the effects of moderate and weak enzyme inhibitors or inducers on the exposures of apatinib have not been

\begin{tabular}{|c|c|c|}
\hline Parameters & Value & Source \\
\hline \multicolumn{3}{|l|}{ Physiochemical parameters } \\
\hline $\log P$ & 3.14 & ALOGPS \\
\hline Compound type & Dibasic base & \\
\hline $\mathrm{pKa}$ & $\mathrm{pKa}_{1}=6.60 \mathrm{pKa}_{2}=5.31$ & In-house data \\
\hline \multicolumn{3}{|l|}{ Absorption parameters } \\
\hline \multicolumn{3}{|l|}{ ADAM model } \\
\hline Caco-2 permeability $\left(10^{-6} \mathrm{~cm} / \mathrm{s}\right)$ & 6.81 & In-house data \\
\hline$P_{\text {eff }}\left(10^{-4} \mathrm{~cm} / \mathrm{s}\right)$ & 0.80 & Calculated using Simcyp \\
\hline \multicolumn{3}{|l|}{ Disposition parameters } \\
\hline Full PBPK model & & Poulin and Theil method \\
\hline Enzyme & CYP 2D6 & Ding et al. (2013) \\
\hline $\mathrm{V}_{\max }(\mathrm{pmol} / \mathrm{min} / \mathrm{mg}$ protein) & 9.82 & \\
\hline $\mathrm{K}_{\mathrm{m}}(\mu \mathrm{M})$ & 1.41 & \\
\hline Enzyme & CYP $3 A 4$ & \\
\hline $\mathrm{V}_{\max }(\mathrm{pmol} / \mathrm{min} / \mathrm{mg}$ protein) & 39.1 & \\
\hline $\mathrm{K}_{\mathrm{m}}(\mu \mathrm{M})$ & 2.18 & \\
\hline Enzyme & CYP $3 A 5$ & \\
\hline $\mathrm{V}_{\max }(\mathrm{pmol} / \mathrm{min} / \mathrm{mg}$ protein) & 3.28 & \\
\hline $\mathrm{K}_{\mathrm{m}}(\mu \mathrm{M})$ & 1.93 & \\
\hline
\end{tabular}


TABLE 2 | Summary of the outline of the trial design for apatinib DDI simulation

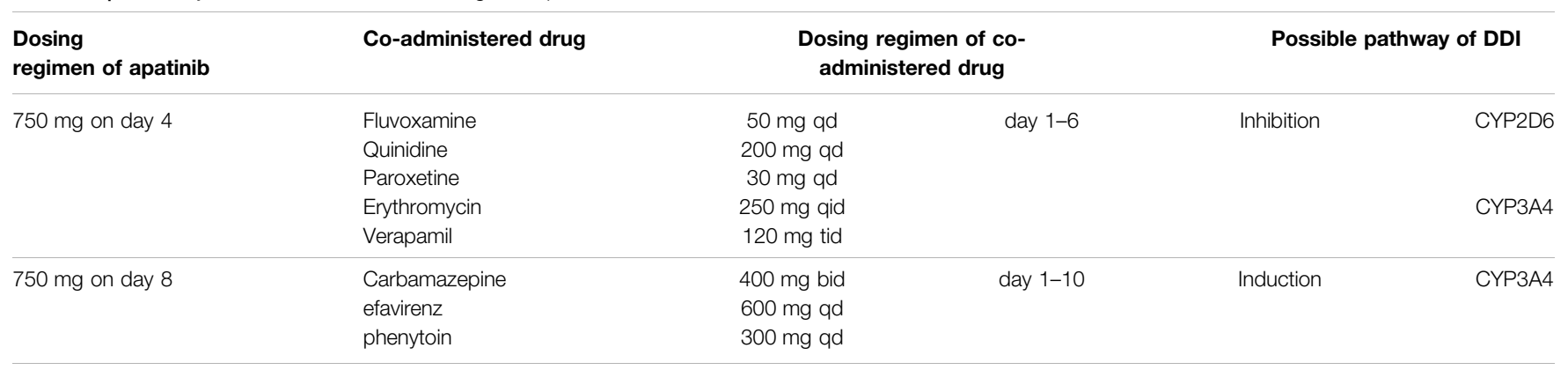

evaluated since cancer patients normally receive other drugs, i.e., hypoglycemics, antihypertensive, anticoagulants, and antifungal, many of which are enzyme inhibitors or inducers. Further research is needed to evaluate its potential involvement in DDIs for the rational clinical administration. In addition, the potential risk assessments of apatinib's pharmacokinetic changes in RIs or HIs will also be of great significance to recommend appropriate dosing regimens.

PBPK modeling is a mathematical predictive approach, which can quantitatively predict ADME process of chemical substances in human or animal by extrapolating in vitro, in situ, and in silico drug-dependent parameters (Min and Bae, 2017; Wu et al., 2020). Some excellent researches and reviews have illustrated the ability of PBPK modeling in drug development (Li et al., 2021; Nauwelaerts et al., 2021; Peng et al., 2021). Hence, a growing emphasis on the application of PBPK modeling has been placed to quantitatively evaluate the potential risks of DDI and DDZI of drugs in vivo (Ono et al., 2017; Rasool et al., 2017; Rasool et al., 2021; Rasool and Läer, 2021). Accordingly, developing a PBPK model of apatinib to quantitatively assess the influence of external and internal factors on apatinib pharmacokinetics would be of great benefit. In the present research, we aimed to establish and verify PBPK models of apatinib on the basis of the reported clinical trials, then the verified PBPK model was applied to evaluate the potential DDI risks with CYP3A4 and CYP2D6 inhibitors or inducers, as well as the DDZI outcomes in HIs and RIs. We focused on gaining insights into the rational clinical administration of apatinib by using the dynamic modeling approach.

\section{MATERIALS AND METHODS}

\section{Clinical Pharmacokinetic Studies of Apatinib}

A systematic literature research was conducted to obtain clinical pharmacokinetic data of apatinib. Briefly, the mean plasma concentration-time profiles of apatinib were determined in Chinese advanced colorectal cancer patients $(n=20)$ at an oral dose of $750 \mathrm{mg}$ following single administration (Ding et al., 2013). The reported single-dose DDI studies were conducted in Chinese healthy volunteers $(n=20)$ with coadministration of rifampin and itraconazole (Liu et al., 2018). Subjects either received $750 \mathrm{mg}$ of oral apatinib mesylate alone on day 1 or treated with $600 \mathrm{mg}$ of oral rifampin once daily for 10 continuous days with concomitant administration of $750 \mathrm{mg}$ apatinib on day 6. For DDI studies with itraconazole, each subject received $250 \mathrm{mg}$ oral apatinib mesylate alone on day 1 or 6-day repeated doses of $100 \mathrm{mg}$ oral itraconazole once daily with concomitant administration of $250 \mathrm{mg}$ apatinib on day 4 .

\section{Development of a Dynamic PBPK Model for Apatinib}

All simulations were conducted using a commercially available PBPK software, Simcyp Simulator (version 16; Simcyp Limited, Sheffield, UK). The input parameters for apatinib PBPK models are listed in Table 1. The physicochemical parameter of molecular weight was obtained from drugbank, and the oil/water partition coefficient (log $\mathrm{P}$ ) was predicted in ALOGPS. The dissociation equilibrium constant $(\mathrm{pKa})$ was determined in our laboratory. Also, plasma unbound fraction $\left(f_{u}\right)$ of apatinib was collected from literature (Ding et al., 2013). The blood to plasma partition coefficient (B/P) was calculated using Simcyp Simulator. The in vivo absorption of apatinib was determined using the Advanced Dissolution Absorption and Transit (ADAM) models, in which the gastrointestinal tract was divided into nine successive compartments. A drug could exist in several states simultaneously within each compartment. The mainly involved parameters in the ADAM setting were permeability and dissolution data. Caco-2 permeability data were used to calculate the effective permeability $\left(\mathrm{P}_{\text {eff }}\right)$ of apatinib in humans. The in vitro dissolution profile of apatinib mesylate tablet was loaded to describe the in vivo release of the drug. The tissue to plasma partition coefficients $\left(\mathrm{K}_{\mathrm{p}}\right)$ of apatinib for all the major tissues in Simcyp Simulator were calculated using Poulin and Theil method with a $\mathrm{K}_{\mathrm{p}}$ scalar of 0.7 . The volume of distribution at steady state $\left(\mathrm{V}_{\mathrm{ss}}\right)$ was predicted using physicochemical properties and $\mathrm{K}_{\mathrm{p}}$. Apatinib was reported to be extensively metabolized via CYP3A4/5 and CYP2D6 (Ding et al., 2013). The enzyme kinetics was used to describe the in vivo clearance of apatinib. The maximum velocity $\left(\mathrm{V}_{\max }\right)$ and Michaelis-Menten constant $\left(\mathrm{K}_{\mathrm{m}}\right)$ were obtained from the reported metabolism and pharmacokinetics of apatinib in humans (Ding et al., 2013).

\section{In vitro Permeability Experiment}

Caco- 2 cells were cultured in Dulbecco's Modified Eagle Medium (DMEM), which contained $10 \%$ fetal bovine serum, $100 \mathrm{U} / \mathrm{ml}$ penicillin G, $100 \mathrm{mg} / \mathrm{ml}$ streptomycin, $1 \%$ nonessential amino acids, and $2 \mathrm{mM}$ glutamine, at the atmosphere of $37^{\circ} \mathrm{C}$ and $5 \%$ 

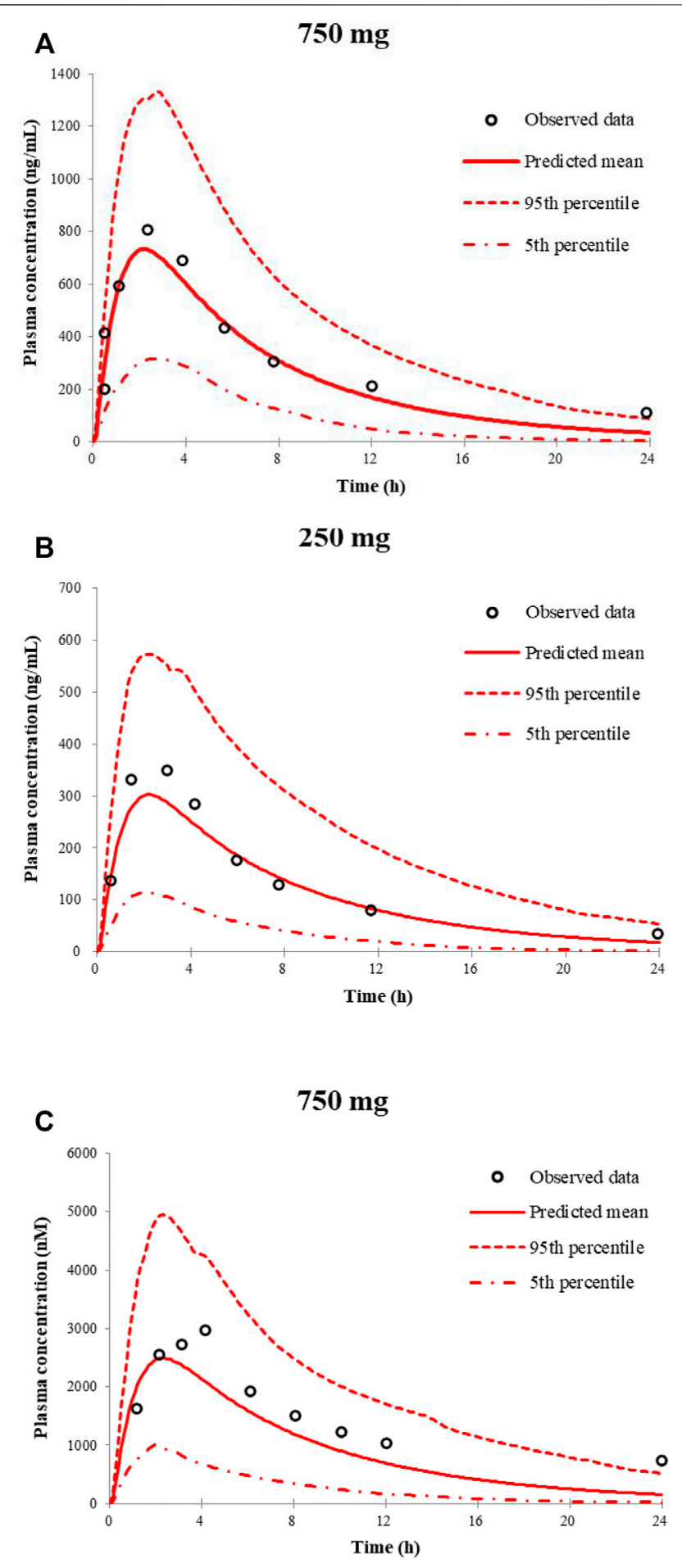

FIGURE 1 | Predicted and observed mean plasma concentration-time curves of apatinib following a single oral dose of apatinib mesylate tablet in healthy volunteers $(\mathbf{A}, \mathbf{B})$ and in cancer patients $(\mathbf{C})$.

$\mathrm{CO}_{2}$. Caco-2 cells in logarithmic growth phase were seeded at a density of $4 \times 10^{4} /$ well onto a 24 Transwell plate. The DMEM was added at a volume of $200 \mu \mathrm{l}$ for apical (AP) compartments and $1,300 \mu \mathrm{l}$ for basolateral (BL) compartments, and changed every other day for 21 days. Then the cell layer was washed three times with pre-heated HBSS buffer, and the transepithelial electrical resistance (TEER) values were measured. Caco-2 monolayers with TEER values $>350 \Omega \cdot \mathrm{cm}^{-2}$ were used for permeability test.

The drug solutions of apatinib mesylate $(10.0 \mu \mathrm{M})$, atenolol $(10.0 \mu \mathrm{M})$, propranolol $(10.0 \mu \mathrm{M})$, and digoxin $(20.0 \mu \mathrm{M})$ were prepared with HBSS buffer consisting of $0.5 \%$ BSA. The $\mathrm{pH}$ was adjusted to 7.4 with $1 \mathrm{M} \mathrm{NaOH}$. The Caco-2 monolayers were rinsed with Hank's balanced salt solution before the experiment and were incubated at $37^{\circ} \mathrm{C}$ for $1 \mathrm{~h}$. Then, the tested compound was added to the $\mathrm{AL}$ or $\mathrm{BL}$ compartments of the polycarboxylate membrane, and the cell culture plate was placed at the atmosphere of $37^{\circ} \mathrm{C}$ and $5 \% \mathrm{CO}_{2}$. After $2 \mathrm{~h}$ of circular vibration culture, samples at a volume of $150 \mu \mathrm{l}$ were taken from the $\mathrm{AL}$ and the $\mathrm{BL}$ compartments. The $\mathrm{P}_{\text {app }}$ values of compounds from $\mathrm{AL}$ to $\mathrm{BL}$ compartments and $\mathrm{BL}$ to $\mathrm{AL}$ compartments were calculated by the following formula:

$$
\mathrm{P}_{\mathrm{app}}=\frac{\triangle \mathrm{Q}}{\triangle T \times A \times C_{0}}
$$

$A$ is the area of the polycarboxylate membrane and $C_{0}$ is the initial concentration.

\section{Verification and Evaluation the Performance of PBPK Model}

The pharmacokinetics of apatinib was simulated via the dynamic PBPK model. The characteristics of subjects and trial design adhered to the clinically reported study, and the simulated results were compared with the observed data (Liu et al., 2018). The fold error of the main pharmacokinetic parameters $\left(\mathrm{C}_{\max }, \mathrm{T}_{\max }\right.$, and AUC) was used to assess the predictive accuracy, which referred to the ratio of the simulated to the observed values (Eq. 1). A desired fold error was between 0.5 and 2.0 (Zhang et al., 2018; Li et al., 2019).

$$
\text { fold error }=\frac{\text { simulated }}{\text { observed }}
$$

For verification of the apatinib DDI models, the DDI predictions of apatinib were performed with itraconazole (strong CYP3A4 inhibitor) and rifampin (strong CYP3A4 inducer). The characteristics of subjects and trial design were based on the clinical study, and the simulated results were compared with the observed data (Liu et al., 2018). The change of apatinib exposure was determined by AUC ratio and $\mathrm{C}_{\max }$ ratio, which referred to the mean $\mathrm{AUC}$ and $\mathrm{C}_{\max }$ in the presence to the absence of inhibitor or inducer, respectively (Eq. 2). The current consensus for successful DDI simulation is that the predicted AUC ratio or $\mathrm{C}_{\max }$ ratio should be less than 2fold (Zhang et al., 2018; Li et al., 2019).

$$
\begin{gathered}
\text { AUC ratio }=\frac{\text { AUC with inhibitor or inducer }}{\text { AUC without inhibitor or inducer }} \\
\mathrm{C}_{\max } \text { ratio }=\frac{\mathrm{C}_{\max } \text { with inhibitor or inducer }}{\mathrm{C}_{\max } \text { without inhibitor or induce }}
\end{gathered}
$$


TABLE 3 | Comparison of model-predicted main pharmacokinetic parameters $\left(C_{\max }, T_{\max }\right.$, and AUC) to the observed data of apatinib mesylate tablet

\begin{tabular}{|c|c|c|c|c|c|}
\hline Dose level & Parameters & Unit & Predicted & Observed & Fold error \\
\hline \multirow[t]{3}{*}{750 mg (in Chinese healthy volunteers) } & $\mathrm{T}_{\max }$ & $\mathrm{h}$ & 2.16 & 3 & 0.72 \\
\hline & $\mathrm{C}_{\max }$ & $\mathrm{ng} / \mathrm{ml}$ & 734.76 & 681 & 1.08 \\
\hline & AUC & $\mathrm{ng} / \mathrm{ml} \cdot \mathrm{h}$ & $5,994.63$ & 7,150 & 0.84 \\
\hline \multirow[t]{3}{*}{250 mg (in Chinese healthy volunteers) } & $\mathrm{T}_{\max }$ & $\mathrm{h}$ & 2.16 & 1.5 & 1.44 \\
\hline & $\mathrm{C}_{\max }$ & $\mathrm{ng} / \mathrm{ml}$ & 302.47 & 371 & 0.82 \\
\hline & $A \cup C$ & $\mathrm{ng} / \mathrm{ml} \cdot \mathrm{h}$ & $2,626.4$ & 2,940 & 0.89 \\
\hline \multirow[t]{3}{*}{750 mg (in Chinese cancer patients) } & $\mathrm{T}_{\max }$ & $\mathrm{h}$ & 2.28 & 2.9 & 0.79 \\
\hline & $\mathrm{C}_{\max }$ & $\mathrm{nM}$ & $2,497.76$ & 3,819 & 0.65 \\
\hline & $A \cup C$ & $\mathrm{nM} \cdot \mathrm{h}$ & $22,274.01$ & 30,941 & 0.72 \\
\hline
\end{tabular}

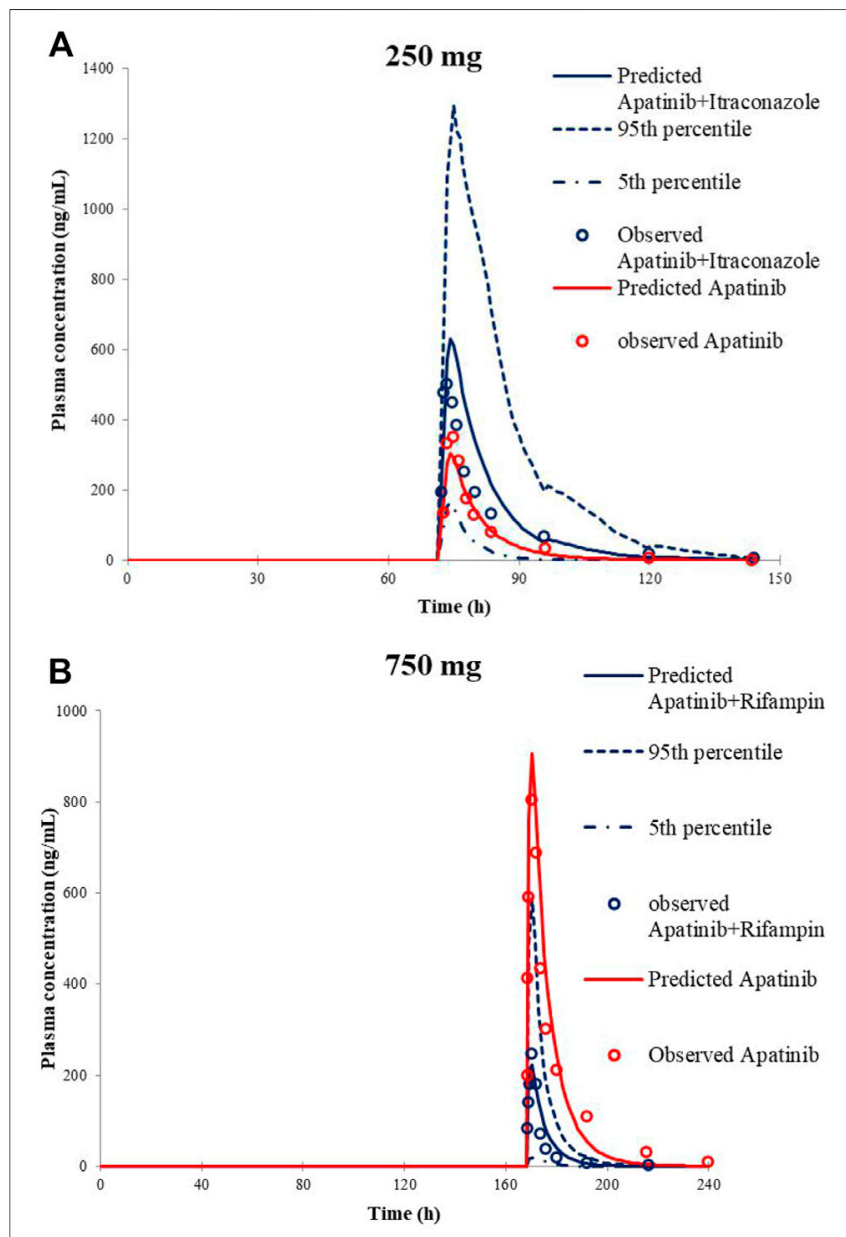

FIGURE 2 | Predicted and observed mean plasma concentration-time curves of apatinib following $250 \mathrm{mg}$ apatinib mesylate tablet in the presence or absence of itraconazole (A) and $750 \mathrm{mg}$ apatinib mesylate tablet in the presence or absence of rifampin (B).

\section{Application of the PBPK Models for Evaluating the Influence of Co-Administered Possible Inhibitors and Inducers on Apatinib Exposure}

The PBPK models were used to predict potential DDI risks with CYP2D6 and CYP3A4 perpetrators. For apatinib DDI simulation with moderate and strong CYP2D6 inhibitors, fluvoxamine $(50 \mathrm{mg}$ once daily (qd)), quinidine (200 $\mathrm{mg} \mathrm{qd}$ ), and paroxetine ( $30 \mathrm{mg} \mathrm{qd}$ ) were used for the PBPK modeling. Erythromycin $(250 \mathrm{mg}$ four times a day (qid)) and verapamil (120 mg three times a day (tid)) were used for apatinib DDI simulation with moderate and weak CYP3A4 inhibitors. For apatinib DDI simulation with CYP3A4 inducers, carbamazepine ( $400 \mathrm{mg}$ twice daily (bid)), efavirenz $(600 \mathrm{mg} \mathrm{qd})$, and phenytoin (300 $\mathrm{mg} \mathrm{qd})$ were used for the PBPK modeling.

Simulation of all DDI outcomes were performed using Simcyp Chinese healthy volunteers in a fasted state with 100 subjects (10 trials $\times 10$ subjects). The outline of the trial design is summarized in Table 2.

\section{Application of the PBPK Models for Predicting the Pharmacokinetic Changes in HIs and RIs}

A single-dose DDZI potential of apatinib in HIs and RIs was evaluated by the PBPK models. The simulation was conducted in a fasted state with 100 subjects (10 trials $\times 10$ subjects) receiving an oral dose of $750 \mathrm{mg}$ apatinib. Virtual populations with Child-Pugh scores A, B, and $\mathrm{C}$ in Simcyp in-built population library were used for simulation in mild, moderate, and severe HIs, respectively. The Simcyp in-built moderate (glomerular filtration rates (GFR) of $30-60 \mathrm{ml} / \mathrm{min}$ ) and severe RI (GFR $<30 \mathrm{ml} / \mathrm{min}$ ) populations were used for simulation in RIs. The pathophysiological changes incorporated within the disease populations used for performing simulations were reduced liver

TABLE 4 | Comparison of model-predicted $\mathrm{C}_{\max }$ and $\mathrm{AUC}$ to the observed one in the presence or the absence of inhibitor or inducer

\begin{tabular}{|c|c|c|c|c|c|c|}
\hline \multirow[t]{2}{*}{ Co-administered drugs } & \multicolumn{2}{|c|}{ Simulated } & \multicolumn{2}{|c|}{ Observed } & \multirow[t]{2}{*}{$\mathrm{C}_{\max }$ ratio $_{\mathrm{s}} / \mathrm{C}_{\max }$ ratio。 } & \multirow[t]{2}{*}{ AUC ratios/AUC ratio。 } \\
\hline & $\mathrm{C}_{\max }$ ratio & AUC ratio & $\mathrm{C}_{\max }$ ratio & AUC ratio & & \\
\hline With itraconazole & 2.08 & 2.69 & 1.34 & 1.71 & 1.55 & 1.57 \\
\hline
\end{tabular}




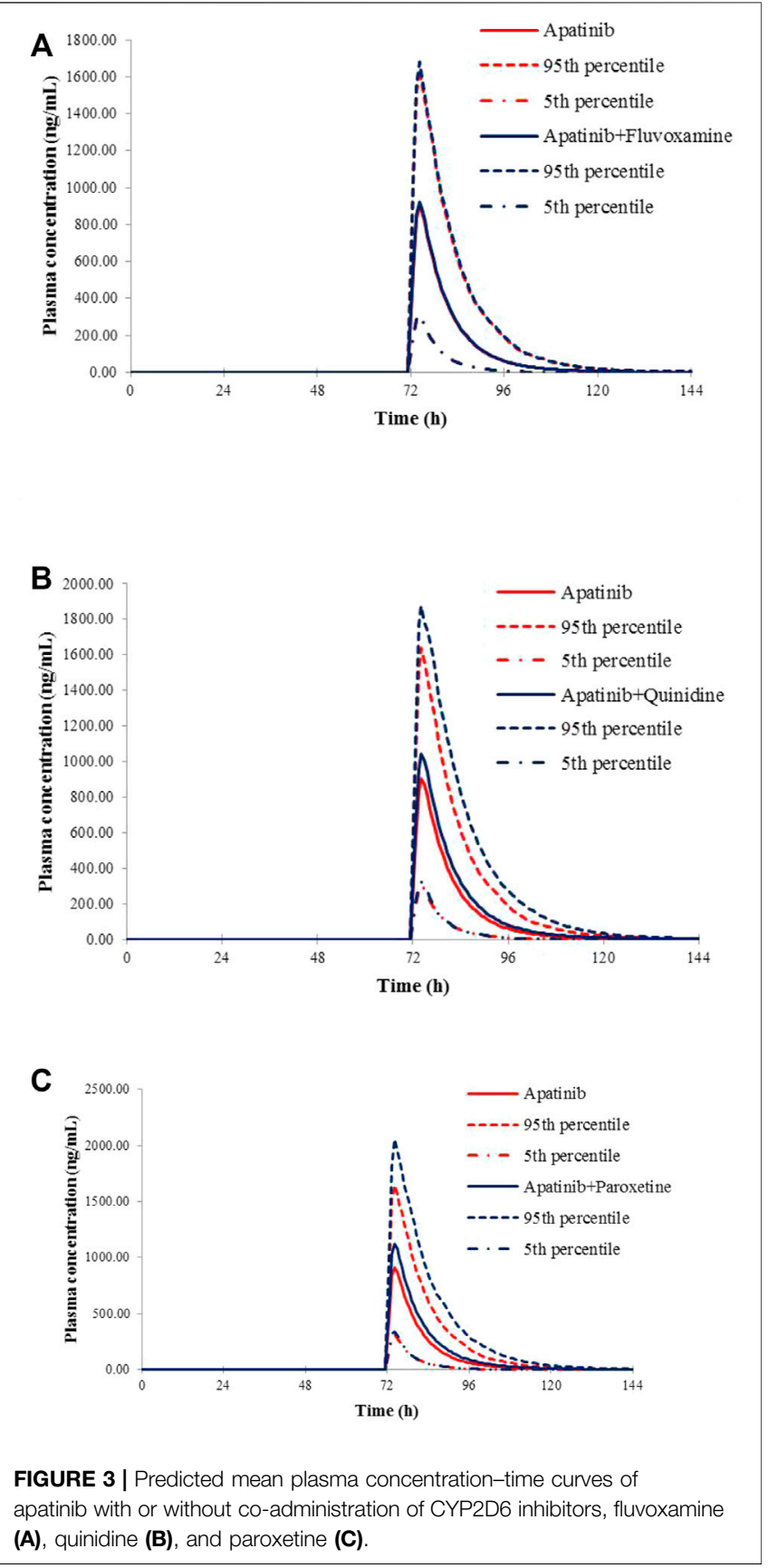

size and kidney weight, reduced hepatic CYP expression (e.g., CYP1A2, 2C9, 2C19, 2D6, and 3A4), reduced serum albumin, $\alpha-1$ acid glycoprotein levels as well as hematocrit levels, altered blood flow, and so on (Ono et al., 2017).

\section{RESULTS}

\section{Verification of the Dynamic PBPK Model for Apatinib}

Figure 1 shows the simulated and observed mean plasma concentration-time curves of apatinib at 750 and $250 \mathrm{mg}$ dose levels in healthy volunteers, as well as the comparison of modelpredicted $750 \mathrm{mg}$ dose level in Chinese healthy volunteers and the observed $750 \mathrm{mg}$ dose level in cancer patients. The comparison of the model-predicted main pharmacokinetic parameters $\left(C_{\max }\right.$, $\mathrm{T}_{\max }$, and AUC) with the observed data and the calculated fold errors for the main pharmacokinetic parameters of apatinib are summarized in Table 3. The predicted mean plasma concentration-time curves of apatinib matched well with the clinically observed one. All the fold errors of $\mathrm{C}_{\max }, \mathrm{T}_{\max }$, and AUC were between 0.5 and 2.0 of the observed data, indicating a good prediction of the apatinib PBPK model. The modelpredicted main pharmacokinetic parameters of apatinib in Chinese healthy volunteers were comparable with the observed one in cancer patients.

\section{Evaluation of the DDI Prediction for Apatinib PBPK Model}

The predicted along with observed mean plasma concentration-time curves of apatinib with co-administered itraconazole or rifampin are described in Figure 2. Table 4 shows the predicted and observed changes of apatinib exposure in the presence of strong inhibitor or inducer of CYP3A4, and calculated AUC ratio as well as $\mathrm{C}_{\max }$ ratio in the presence to the absence of inhibitor or inducer, respectively. On the basis of the results, the developed PBPK model successfully simulated the DDIs of apatinib with itraconazole, and rifampin. Thus, investigation of the DDIs of apatinib could be conducted by the verified PBPK models.

\section{Application of the Apatinib PBPK Model for the Evaluation of the Potential DDI Risks}

The verified PBPK models of apatinib were applied to evaluate the potential DDI risks with moderate and weak CYP3A4 inhibitors, strong CYP2D6 inhibitors, and the CYP3A4 inducers at therapeutic doses. The trial design was based on the clinical reports shown in Table 2 . The DDI outcomes are depicted in Figures 3-5, and the calculated ratio of $C_{\max }$ or AUC is shown in Table 5. The exposure change $\left(C_{\max }\right.$ or AUC) by over 2 -fold was considered to be significant in the DDI simulations. In this context, the exposure of apatinib was obviously affected by coadministration of erythromycin, verapamil, carbamazepine, efavirenz, and phenytoin, respectively. A moderate increase of apatinib exposure $\left(\mathrm{C}_{\max }\right.$ or AUC ratio between 1.25 and 2.0) was found with paroxetine. It appeared that moderate CYP2D6 inhibitor fluvoxamine and quinidine had negligible influence on the exposure of apatinib at therapeutic dose.

\section{Application of the Apatinib PBPK Model for Evaluation of the Potential DDZI Risks}

The virtual populations of Chinese healthy volunteers (HVs), HIs with Child-Pugh A, B, or C, and moderate and severe RIs in Simcyp in-built population library received an oral dose of $750 \mathrm{mg}$ apatinib mesylate tablet. The simulated mean plasma 




concentration-time curves are depicted in Figure 6. As shown in Table 6, the predicted $C_{\max }$ ratio and AUC ratio were 1.02 and 1.09 for Child-Pugh A, 1.54 and 2.25 for Child-Pugh B, and 1.67 and 3.04 for Child-Pugh C, respectively, in HIs. The predicted $\mathrm{C}_{\max }$ ratio and AUC ratio were 1.02 and 1.06 for moderate RIs, and 1.11 and 1.01 for severe RIs, respectively. The impact of RIs was negligible on the exposure of apatinib at therapeutic dose.

\section{DISCUSSION}

In the present study, we first developed a PBPK model for apatinib by incorporating both in vitro and in vivo data. The pharmacokinetics of apatinib at an oral dose level of 750 and $250 \mathrm{mg}$ in healthy volunteers, as well as $750 \mathrm{mg}$ dose level in cancer patients, were predicted. The results showed that the pharmacokinetic profiles of apatinib matched well with the clinically observed one. Baker et al. have reported that age, sex, and body size have negligible influence on the CYP3A activity in 134 cancer patients (Baker et al., 2004). Consistently, midazolam, a CYP3A probe substrate, was predicted to have comparable exposure between healthy volunteers and cancer patients using PBPK modeling (Cheeti et al., 2013). The PBPK model developed by Chiho et al. captured comparable pharmacokinetics of bosutinib in healthy volunteers with cancer patients as well (Ono et al., 2017). Therefore, the Simcyp default "Chinese Healthy Volunteers" population file was

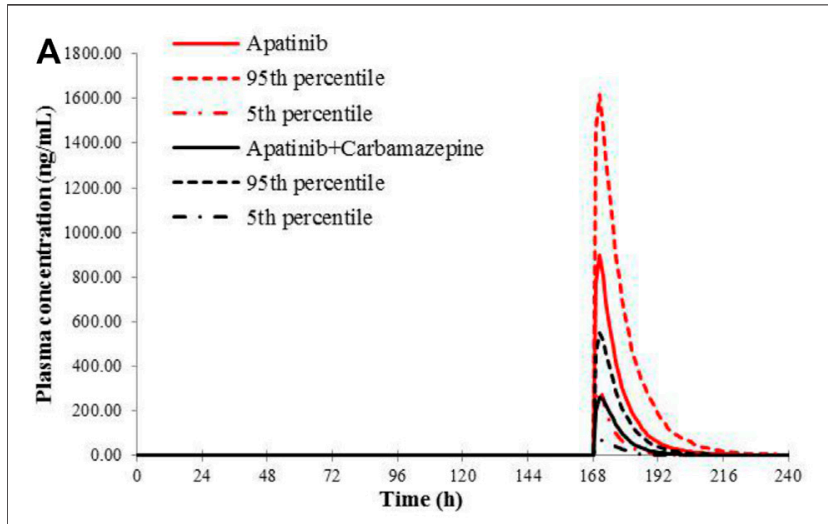

B


FIGURE 5 | Predicted mean plasma concentration-time curves of apatinib with or without co-administration of CYP 3A4 inducers, carbamazepine (A), efavirenz (B), and phenytoin (C).

used both in simulation with healthy volunteers and cancer patients. Our study also demonstrated the similar simulation in these two populations. Afterwards, the validation of the apatinib DDI model was conducted using the reported clinical study, which showed the successful simulation of the exposure changes of apatinib in the presence or absence of itraconazole and rifampicin (Liu et al., 2018).

Then, the potential risks of DDI and DDZI of apatinib were estimated using the verified PBPK model. Owing to that cancer patients are frequently treated with multiple medications, the 
TABLE 5 | Simulated exposure changes of apatinib with co-administration of possible inhibitors or inducers

\begin{tabular}{|c|c|c|c|c|c|}
\hline \multirow{3}{*}{$\begin{array}{l}\text { Dosage } \\
\text { regime of apatinib } \\
750 \mathrm{mg} \text { on day } 4\end{array}$} & \multirow{3}{*}{$\begin{array}{l}\text { Co-administered inhibitors or } \\
\text { inducers }\end{array}$} & \multirow{2}{*}{\multicolumn{2}{|c|}{$\begin{array}{l}\text { Dosage regime of co- } \\
\text { administered drugs }\end{array}$}} & \multicolumn{2}{|c|}{ Simulated } \\
\hline & & & & \multirow{2}{*}{$\begin{array}{c}\mathbf{C}_{\max } \text { ratio } \\
1.02\end{array}$} & \multirow{2}{*}{$\begin{array}{c}\text { AUC ratio } \\
1.02\end{array}$} \\
\hline & & 50 mg qd & Days 1-6 & & \\
\hline & Quinidine & $200 \mathrm{mg}$ qd & & 1.15 & 1.21 \\
\hline & Paroxetine & 30 mg qd & & 1.23 & 1.29 \\
\hline & Erythromycin & $250 \mathrm{mg}$ qid & & 2.23 & 3.60 \\
\hline & Verapamil & 120 mg tid & & 1.76 & 2.17 \\
\hline \multirow[t]{3}{*}{$750 \mathrm{mg}$ on day 8} & Carbamazepine & 400 mg bid & Days $1-10$ & 0.29 & 0.30 \\
\hline & Efavirenz & $600 \mathrm{mg}$ qd & & 0.45 & 0.36 \\
\hline & Phenytoin & $300 \mathrm{mg}$ qd & & 0.37 & 0.34 \\
\hline
\end{tabular}

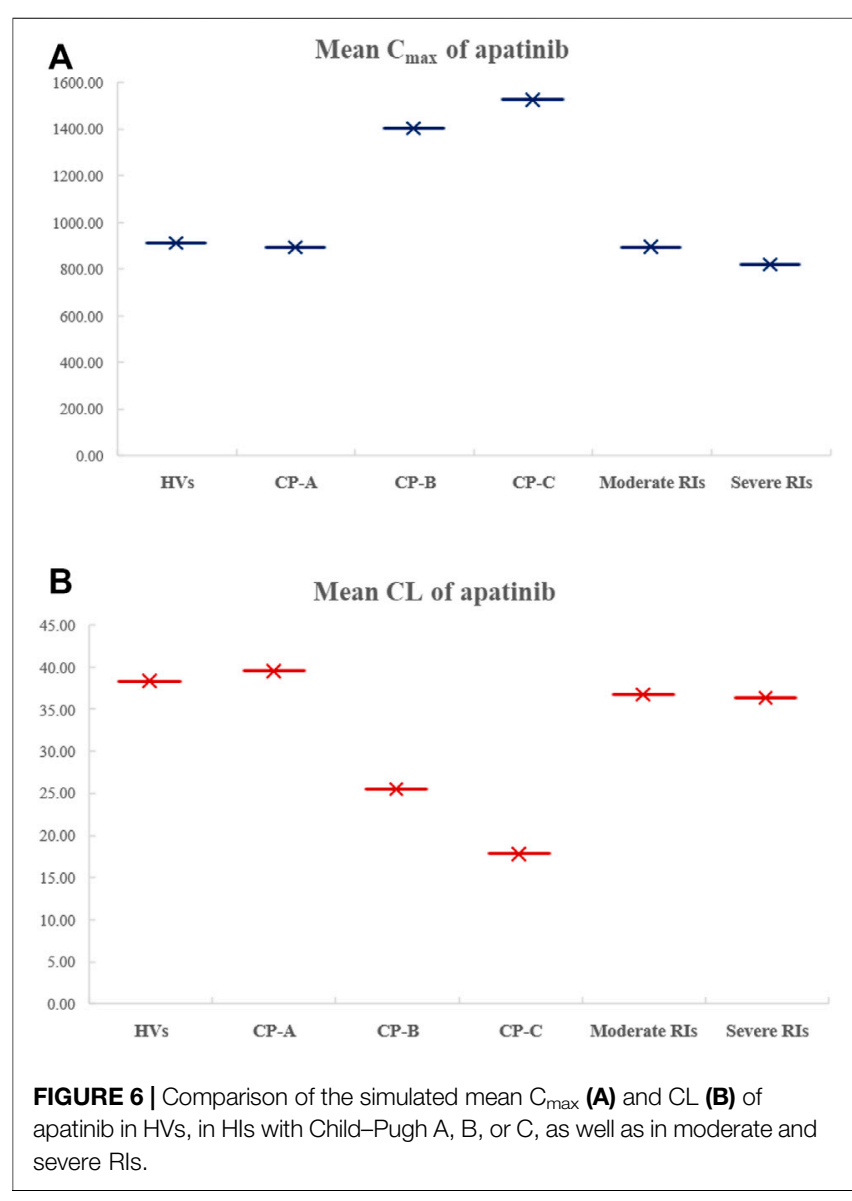

TABLE 6 | Simulated exposure changes of apatinib in patients with $\mathrm{HI}$ and $\mathrm{RI}$

\begin{tabular}{lcccc}
\hline Subjects & $\mathbf{C}_{\max }(\mathbf{n g} / \mathbf{m l})$ & AUC $(\mathbf{n g} / \mathbf{m l} \cdot \mathbf{h})$ & \multicolumn{2}{c}{ Ratio } \\
\cline { 4 - 5 } & & & $\mathbf{R}_{\mathbf{C m a x}}$ & $\mathbf{R}_{\text {AUc }}$ \\
\hline HVs & 911.56 & $7,934.66$ & & \\
HI CP-A & 892.54 & $8,641.86$ & 1.02 & 1.09 \\
HI CP-B & $1,402.36$ & $17,880.77$ & 1.54 & 2.25 \\
HI CP-C & $1,524.33$ & $24,141.57$ & 1.67 & 3.04 \\
Moderate Rls & 894.98 & $8,449.86$ & 1.02 & 1.06 \\
Severe Rls & 820.29 & $8,050.40$ & 1.11 & 1.01
\end{tabular}

potential DDI risks of apatinib co-administered with other drugs must be determined. Apatinib was reported to be metabolized primarily via CYP3A4/5 and CYP2D6 (Ding et al., 2013). Therefore, the verified PBPK model was applied to evaluate the potential DDI risks involving CYP3A4 and CYP2D6mediated metabolizing pathways. Erythromycin and verapamil were moderate CYP3A4 inhibitors (Zhou, 2008; Yamazaki et al., 2015). In our simulations, apatinib exposure was obviously increased in subjects concomitantly treated with erythromycin and verapamil. The predicted AUC of DDI ratio was 3.60 and 2.17 with co-administration of erythromycin and verapamil, respectively. The presence of paroxetine, a strong CYP2D6 inhibitor, could also lead to a moderate increase of the exposure of apatinib by 1.29 -fold. The simulated result was also consistent with the fact that the metabolism of apatinib via CYP3A4 was much higher than that of CYP2D6. Thus, attention should be paid to the co-administration of apatinib with moderate CYP3A4 inhibitors and the corresponding dose adjustment in clinic. In the DDI simulation with CYP3A4 inducers, the predicted apatinib exposure decreased by $71.8,61.6$, and $63.9 \%$ in the presence of carbamazepine, efavirenz, and phenytoin, respectively, indicating that we should be careful about the pharmacokinetic interactions of apatinib with CYP3A4 inducers, which is likely to impair the efficacy.

It has been reported that cirrhosis could decrease gastrointestinal absorption and reduce the expression of drug-metabolizing enzymes as well as transporters (Verbeeck, 2008). The renal impairment could cause decreases in renal clearance along with other changes such as activity of drugmetabolizing enzyme, especially in CYP3A4 and reduced plasma protein binding (Yeung et al., 2014). Thus, the DDZI risks should be assessed in HIs and RIs for appropriate dosing recommendations. In the DDZI simulation with HIs, the AUC of apatinib was significantly increased by 2.25 -fold and 3.04 -fold for Child-Pugh B and Child-Pugh C, respectively, with slight but consistent decrease of $\mathrm{C}_{\max }$ by 1.54 - and 1.67-fold, respectively. The results were in accordance with the alteration of absorption and the decrease in CYP enzymemediated clearance of apatinib due to decreased enzyme abundances in patients with HIs. The model-predicted AUC ratios in moderate and severe RIs were 1.06 and 1.01, respectively, suggesting the negligible impact of renal 
impairment on the exposure of apatinib. In view of the challenges to conduct various DDI and DDZI studies in cancer patients, the PBPK model would be of great benefit to quantitatively predict pharmacokinetic changes under different circumstances which might be difficult to evaluate clinically, so as to avoid some DDI and DDZI risks in advance.

However, some limitations in this study warrant further discussion. First, the present study focused on the pharmacokinetic changes of apatinib as a victim drug; the impact of apatinib as perpetrators on other drugs being mainly metabolized via CYP3A4 or CYP2D6 has not been evaluated. It has been demonstrated that apatinib inhibited the metabolism of gefitinib in vitro and in vivo via CYP2D6 and CYP3A4 (Wang et al., 2021). Zhu et al. have investigated the potential of apatinib as a perpetrator on the pharmacokinetics of nifedipine and warfarin in advanced solid tumor patients. The results indicated that concomitant administration of apatinib led to an obvious increase to nifedipine and warfarin exposure (Zhu et al., 2020). Due to the risk of pharmacokinetic DDI based on enzyme inhibition by apatinib, a dynamic PBPK model is needed for further dosing recommendations when apatinib is co-administered with CYP3A4, CYP2D6, or CYP2C9 substrates. Second, we only evaluated the DDZI outcomes at a single dose level; the multiple-dose DDZIs were not incorporated in the simulation, although the comparable changes of exposures in HIs between single- and multipledose administrations were found in some drugs such as bosutinib (Ono et al., 2017). Third, the model-predicted impacts of HIs and RIs on the pharmacokinetics of apatinib required further clinical verification, considering potential physiological changes resulted from impaired hepatic or renal function. There exist considerable challenges to accurately incorporate the complex physiological changes into PBPK models due to the changes of hepatic architecture in HIs. As a result, bosutinib exposures could not be well predicted in HIs compared with the clinical observed data by Chiho et al. (Ono et al., 2017). The apatinib PBPK model is expected to be further refined using the post-marketing observed data in HIs and RIs, and therefore more adequately evaluating the potential DDZI risks.

All in all, the present study showed that the verified PBPK model of apatinib could provide mechanistic insights for further understanding the potential risk of DDIs and DDZIs, providing the reasonable dosing recommendations.

\section{CONCLUSION}

In the present study, we have developed and verified a dynamic PBPK model of apatinib based on the currently available data.
The models can be used to predict apatinib exposures in the single-dose DDIs with other CYP inhibitors and DDZIs in HIs or RIs. The DDI prediction indicated significant changes (2-4fold) in apatinib exposures with moderate CYP3A4 inhibitors or CYP3A4 inducers. A moderate increase of apatinib exposure (1.25-2-fold) was found with strong CYP2D6 inhibitor. Clinicians should be cautious about the necessary dose adjustment of apatinib being used with these perpetrators. In the DDZI simulation with HIs, the AUC of apatinib was significantly increased by 2.25-fold and 3.04-fold for Child-Pugh B and Child-Pugh $\mathrm{C}$, respectively, with slight but consistent decrease of $\mathrm{C}_{\max }$ by $1.54-$ and 1.67 -fold, respectively. The DDZI prediction suggested a negligible increase in apatinib exposures in RIs. The PBPK models could be of great benefit to give some recommendations in DDI and DDZI risk assessments, and replace some clinical studies as well. The verified PBPK model could provide a rough understanding of the extrinsic and intrinsic factors on the exposure of apatinib.

\section{DATA AVAILABILITY STATEMENT}

The original contributions presented in the study are included in the article/Supplementary Material; further inquiries can be directed to the corresponding authors.

\section{AUTHOR CONTRIBUTIONS}

Participated in research design: HL and XX. Conducted experiments: HL. Performed data analysis: HL and YY. Contributed new reagents or analytic tools: NG and XW. Wrote or contributed to the writing of the article: $\mathrm{HL}, \mathrm{BH}$, and $\mathrm{XX}$.

\section{FUNDING}

This project was supported from a framework of China-Korea international cooperation program managed by National Natural Science Foundation of China and National Research Foundation of Korea (No. 82011540409) and Minhang District Science and Technology Commission Foundation (No. 2020MHZ035).

\section{ACKNOWLEDGMENTS}

Certara United Kingdom (Simcyp Division) granted free access to the Simcyp Simulators through an academic license. Pharmacology Department of Hengrui Co., Ltd helped to the in vitro experimets of apatinib. 


\section{REFERENCES}

Baker, S. D., van Schaik, R. H., Rivory, L. P., Ten Tije, A. J., Dinh, K., Graveland, W. J., et al. (2004). Factors Affecting Cytochrome P-450 3A Activity in Cancer Patients. Clin. Cancer Res. 10 (24), 8341-8350. doi:10.1158/1078-0432.CCR-041371

Cheeti, S., Budha, N. R., Rajan, S., Dresser, M. J., and Jin, J. Y. (2013). A Physiologically Based Pharmacokinetic (PBPK) Approach to Evaluate Pharmacokinetics in Patients with Cancer. Biopharm. Drug Dispos 34 (3), 141-154. doi:10.1002/bdd. 1830

Chung, J., and Kesisoglou, F. (2018). Physiologically Based Oral Absorption Modelling to Study Gut-Level Drug Interactions. J. Pharm. Sci. 107 (1), 18-23. doi:10.1016/j.xphs.2017.08.015

Committee for Human Medicinal Products (2014). Guideline on the Evaluation of the Pharmacokinetics of Medicinal Products in Patients with Decreased Renal Function. Netherland: European Medicines Agency. Available at: http://www.ema.europa.eu/ docs/en_GB/document_library/Scientific_guideline/2014/02/WC500162133.pdf.

Committee for Human Medicinal Products (2012). Guideline on the Investigation of Drug Interactions. Netherland: European Medicines Agency. Available at: http://www.ema.europa.eu/docs/en_GB/ document_library/Scientific_guideline/2012/07/WC500129606.pdf.

Committee for Human Medicinal Products (2015). Guidline on the Evaluation of the Pharmacokinetics of Medicinal Products in Patients with Impaired Hepatic Function. Netherland: European Medicines Agency. Available at: http://www.ema.europa.eu/ docs/en_GB/document_library/Scientific_guideline/2009/09/WC500003122.pdf.

Ding, J., Chen, X., Gao, Z., Dai, X., Li, L., Xie, C., et al. (2013). Metabolism and Pharmacokinetics of Novel Selective Vascular Endothelial Growth Factor Receptor-2 Inhibitor Apatinib in Humans. Drug Metab. Dispos 41 (6), 1195-1210. doi:10.1124/dmd.112.050310

Drug Interaction Studies (2012). Study Design, Data Analysis, Implications for Dosing, and Labeling Recommendations Draft Guidance. The Maryland: US Department of Health and Human Services. Food and Drug Administration, Center for Drug Evaluation and Research. Available at: http://www.fda.gov/downloads/Drugs/ GuidanceComplianceRegulatoryInformation/Guidances/ucm292362.pdf.

Drug Interaction Studies (2014). Study Design, Data Analysis, Implications for Dosing, and Labeling Recommendations Draft Guidance. Tokyo: Pharmaceuticals and Medical Devices Agency. Available at: http://www. japal.org/contents/pdf/notice/jimurenraku/20140708_jimu.pdf.

Fathi Maroufi, N., Rashidi, M. R., Vahedian, V., Akbarzadeh, M., Fattahi, A., and Nouri, M. (2020). Therapeutic Potentials of Apatinib in Cancer Treatment: Possible Mechanisms and Clinical Relevance. Life Sci. 241 (241), 117106. doi:10.1016/ j.lfs.2019.117106

Geng, R., Song, L., Li, J., and Zhao, L. (2018). The Safety of Apatinib for the Treatment of Gastric Cancer. Expert Opin. Drug Saf. 17 (11), 1145-1150. doi:10.1080/14740338.2018.1535592

Guidance for Industry (2003). Pharmacokinetics in Patients with Impaired Hepatic Function: Study Design, Data Analysis, and Impact on Dosing and Labeling. The Maryland: US Department of Health and Human Services. Food and Drug Administration, Center for Drug Evaluation and Research (CDER), Center for Biologics Evaluation and Research. Available at: http://www.fda.gov/ downloads/drugs/guidancecomplianceregulatoryinformation/guid ances/ ucm072123.pdf.

Guidance for Industry (2010). Pharmacokinetics in Patients with Impaired Renal Function-Study Design, Data Analysis, and Impact on Dosing and Labeling. The Maryland: US Department of Health and Human Services. Food and Drug Administration, Center for Drug Evaluation and Research. Available at: http:// www.fda.gov/downloads/Drugs/Guidances/UCM204959.pdf.

Li, S., Yu, Y., Bian, X., Yao, L., Li, M., Lou, Y. R., et al. (2021). Prediction of Oral Hepatotoxic Dose of Natural Products Derived from Traditional Chinese Medicines Based on SVM Classifier and PBPK Modeling. Arch. Toxicol. 95 (5), 1683-1701. doi:10.1007/s00204-021-03023-1

Li, S., Yu, Y., Jin, Z., Dai, Y., Lin, H., Jiao, Z., et al. (2019). Prediction of Pharmacokinetic Drug-Drug Interactions Causing Atorvastatin-Induced Rhabdomyolysis Using Physiologically Based Pharmacokinetic Modelling. Biomed. Pharmacother. 119, 109416. doi:10.1016/ j.biopha.2019.109416
Liu, X., Zhang, Y., Chen, Q., Zhan, Y., Wang, Q., Hu, C., et al. (2018). Pharmacokinetic Drug Interactions of Apatinib with Rifampin and Itraconazole. J. Clin. Pharmacol. 58 (3), 347-356. doi:10.1002/jcph.1016

Meng, X., Wang, H., Zhao, J., Hu, L., Zhi, J., Wei, S., et al. (2020). Apatinib Inhibits Cell Proliferation and Induces Autophagy in Human Papillary Thyroid Carcinoma via the PI3K/Akt/mTOR Signaling Pathway. Front. Oncol. 10, 217-229. doi:10.3389/fonc.2020.00217

Min, J. S., and Bae, S. K. (2017). Prediction of Drug-Drug Interaction Potential Using Physiologically Based Pharmacokinetic Modeling. Arch. Pharm. Res. 40 (12), 1356-1379. doi:10.1007/s12272-017-0976-0

Nauwelaerts, N., Deferm, N., Smits, A., Bernardini, C., Lammens, B., Gandia, P., et al. (2021). A Comprehensive Review on Non-clinical Methods to Study Transfer of Medication into Breast Milk - A Contribution from the ConcePTION Project. Biomed. Pharmacother. 136, 111038. doi:10.1016/ j.biopha.2020.111038

Ono, C., Hsyu, P. H., Abbas, R., Loi, C. M., and Yamazaki, S. (2017). Application of Physiologically Based Pharmacokinetic Modeling to the Understanding of Bosutinib Pharmacokinetics: Prediction of Drug-Drug and Drug-Disease Interactions. Drug Metab. Dispos 45 (4), 390-398. doi:10.1124/dmd.116.074450

Peng, Y., Cheng, Z., and Xie, F. (2021). Evaluation of Pharmacokinetic Drug-Drug Interactions: a Review of the Mechanisms, In Vitro and In Silico Approaches. Metabolites 11 (2), 75-90. doi:10.3390/metabo11020075

Rasool, M. F., Ali, S., Khalid, S., Khalid, R., Majeed, A., Imran, I., et al. (2021) Development and Evaluation of Physiologically Based Pharmacokinetic DrugDisease Models for Predicting Captopril Pharmacokinetics in Chronic Diseases. Sci. Rep. 11 (1), 8589-8604. doi:10.1038/s41598-021-88154-2

Rasool, M. F., Khalil, F., and Läer, S. (2017). Optimizing the Clinical Use of Carvedilol in Liver Cirrhosis Using a Physiologically Based Pharmacokinetic Modeling Approach. Eur. J. Drug Metab. Pharmacokinet. 42 (3), 383-396. doi:10.1007/s13318-016-0353-2

Rasool, M. F., and Läer, S. (2021). Development and Evaluation of a Physiologically Based Pharmacokinetic Model to Predict CarvedilolParoxetine Metabolic Drug-Drug Interaction in Healthy Adults and its Extrapolation to Virtual Chronic Heart Failure Patients for Dose Optimization. Expert Opin. Drug Metab. Toxicol. 17, 717-724. doi:10.1080/17425255.2021.1921145

Rekić, D., Reynolds, K. S., Zhao, P., Zhang, L., Yoshida, K., Sachar, M., et al. (2017). Clinical Drug-Drug Interaction Evaluations to Inform Drug Use and Enable Drug Access. J. Pharm. Sci. 106 (9), 2214-2218. doi:10.1016/ j.xphs.2017.04.016

Roviello, G., Ravelli, A., Fiaschi, A. I., Cappelletti, M. R., Gobbi, A., Senti, C., et al. (2016). Apatinib for the Treatment of Gastric Cancer. Expert Rev. Gastroenterol. Hepatol. 10 (8), 887-892. doi:10.1080/17474124.2016.1209407

Verbeeck, R. K. (2008). Pharmacokinetics and Dosage Adjustment in Patients with Hepatic Dysfunction. Eur. J. Clin. Pharmacol. 64 (12), 1147-1161. doi:10.1007/ s00228-008-0553-Z

Wang, C., Ying, L., Jin, M., Zhang, F., Shi, D., Dai, Y., et al. (2021). An Investigation into Possible Interactions Among Four Vascular Epidermal Growth Factor Receptor-Tyrosine Kinase Inhibitors with Gefitinib. Cancer Chemother. Pharmacol. 87 (1), 43-52. doi:10.1007/s00280-020-04191-0

Wu, F., Krishna, G., and Surapaneni, S. (2020). Physiologically Based Pharmacokinetic Modeling to Assess Metabolic Drug-Drug Interaction Risks and Inform the Drug Label for Fedratinib. Cancer Chemother. Pharmacol. 86 (4), 461-473. doi:10.1007/s00280-020-04131-y

Xue, J. M., Astère, M., Zhong, M. X., Lin, H., Shen, J., and Zhu, Y. X. (2018). Efficacy and Safety of Apatinib Treatment for Gastric Cancer, Hepatocellular Carcinoma and Non-small Cell Lung Cancer: A Meta-Analysis. Onco Targets Ther. 11 (11), 6119-6128. doi:10.2147/OTT.S172717

Yamazaki, S., Johnson, T. R., and Smith, B. J. (2015). Prediction of Drug-Drug Interactions with Crizotinib as the CYP3A Substrate Using a Physiologically Based Pharmacokinetic Model. Drug Metab. Dispos 43 (10), 1417-1429. doi:10.1124/dmd.115.064618

Yeung, C. K., Shen, D. D., Thummel, K. E., and Himmelfarb, J. (2014). Effects of Chronic Kidney Disease and Uremia on Hepatic Drug Metabolism and Transport. Kidney Int. 85 (3), 522-528. doi:10.1038/ki.2013.399

Zhang, H. (2015). Apatinib for Molecular Targeted Therapy in Tumor. Drug Des. Devel Ther. 9 (60), 6075-6081. doi:10.2147/DDDT.S97235 
Zhang, H., Bu, F., Li, L., Jiao, Z., Ma, G., Cai, W., et al. (2018). Prediction of DrugDrug Interaction between Tacrolimus and Principal Ingredients of Wuzhi Capsule in Chinese Healthy Volunteers Using Physiologically-Based Pharmacokinetic Modelling. Basic Clin. Pharmacol. Toxicol. 122 (3), 331-340. doi:10.1111/bcpt.12914

Zhang, L., Xu, N., Xiao, S., Arya, V., Zhao, P., Lesko, L. J., et al. (2012). Regulatory Perspectives on Designing Pharmacokinetic Studies and Optimizing Labeling Recommendations for Patients with Chronic Kidney Disease. J. Clin. Pharmacol. 52 (1 Suppl. 1), 79S-90S. doi:10.1177/0091270011415410

Zhou, S. F. (2008). Drugs Behave as Substrates, Inhibitors and Inducers of Human Cytochrome P450 3A4. Curr. Drug Metab. 9 (4), 310-322. doi:10.2174/ 138920008784220664

Zhu, Y. T., Teng, Z., Zhang, Y. F., Li, W., Guo, L. X., Liu, Y. P., et al. (2020). Effects of Apatinib on the Pharmacokinetics of Nifedipine and Warfarin in Patients with Advanced Solid Tumors. Drug Des. Devel Ther. 14, 1963-1970. doi:10.2147/DDDT.S237301
Conflict of Interest: The authors declare that the research was conducted in the absence of any commercial or financial relationships that could be construed as a potential conflict of interest.

Publisher's Note: All claims expressed in this article are solely those of the authors and do not necessarily represent those of their affiliated organizations, or those of the publisher, the editors, and the reviewers. Any product that may be evaluated in this article, or claim that may be made by its manufacturer, is not guaranteed or endorsed by the publisher.

Copyright (C) $2021 \mathrm{Liu}, \mathrm{Yu}, \mathrm{Guo}$, Wang, Han and Xiang. This is an open-access article distributed under the terms of the Creative Commons Attribution License (CC BY). The use, distribution or reproduction in other forums is permitted, provided the original author(s) and the copyright owner(s) are credited and that the original publication in this journal is cited, in accordance with accepted academic practice. No use, distribution or reproduction is permitted which does not comply with these terms. 